\title{
Sampled Grating Tunable Twin-Guide Laser Diodes With Over 40-nm Electronic Tuning Range
}

\author{
René Todt, Thomas Jacke, Ralf Meyer, Jörg Adler, Reinhard Laroy, Geert Morthier, Senior Member, IEEE, and \\ Markus-Christian Amann, Senior Member, IEEE
}

\begin{abstract}
Device and tuning characteristics of sampled grating tunable twin-guide laser diodes are presented. The vertically integrated, monolithic widely tunable laser requires only two tuning currents to fully cover a wavelength range of more than $40 \mathrm{~nm}$ by electrooptic tuning. Its tuning behavior is quasi-continuous with up to 8.2-nm broad continuous tuning regions. High sidemode suppression (SMSR $\geq 35 \mathrm{~dB})$ as well as large output power $(P \geq$ $10 \mathrm{~mW}$ ) are obtained over the wavelength range from 1520.5 to $1561.5 \mathrm{~nm}$.
\end{abstract}

Index Terms-Distributed feedback (DFB), optical communication, semiconductor lasers, tunable lasers, Vernier effect, wavelength tuning.

\section{INTRODUCTION}

W IDELY tunable laser diodes with tuning ranges of several tens of nanometers are key components for optical communication networks [1]. While being crucial for the development of advanced optical networks that, for example, perform switching solely in the optical domain, they additionally offer potential for inventory cost reduction in present dense wavelength-division-multiplexing networks. This requires, however, that the tunable laser performs equally well as the conventional fixed wavelength distributed feedback (DFB) laser and is at the same time only slightly more expensive.

Even though widely tunable lasers have been extensively investigated in recent years (for an overview see [1] and [2]), presently available monolithic devices suffer from complex tuning schemes, requiring three or even more tuning currents to set the emission wavelength. Accordingly, device characterization and control is complicated, time-consuming and, hence, expensive.

Monolithic widely tunable laser diodes can be divided into two classes: distributed Bragg reflector (DBR)- and DFB-type devices. Inherent to all DBR-like lasers [1], [3], [4] is that they require a phase tuning section to adjust the cavity mode location. On the other hand, a phase tuning section is not needed in

\footnotetext{
Manuscript received July 19, 2005; revised August 16, 2005. This work was supported by the European Union via the Information Society Technologies (IST) Project IST-2000-28244 "NEWTON." The work of R. Laroy was supported by the Institute for the Promotion of Innovation by Science and Technology in Flanders (IWT) under a specialization grant.

R. Todt, T. Jacke, R. Meyer, and M.-C. Amann are with the Walter Schottky Institute, Technische Universität München, D-85748 Garching, Germany (e-mail: todt@wsi.tum.de).

J. Adler is with Infineon Technologies Fiber Optics GmbH, D-13629 Berlin, Germany.

R. Laroy and G. Morthier are with the Department of Information Technology, Ghent University, B-9000 Gent, Belgium.

Digital Object Identifier 10.1109/LPT.2005.859155
}

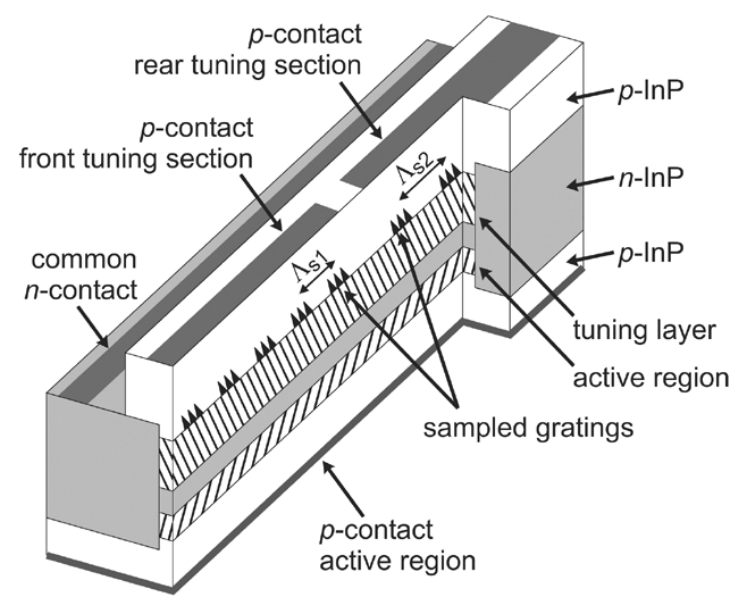

Fig. 1. Schematic drawing of a widely tunable twin-guide laser diode with SGs. For the sake of clarity, the window structures have not been included in the figure.

DFB-like devices [5]-[8]. Hence, they offer potential to facilitate device characterization and control by a reduction of the number of tuning currents, which is desirable as long as the device performance is not impaired.

However, despite this apparent advantage of the DFB-based device concepts, only little experimental data has been presented to date. Decent performance has been obtained from thermally widely tunable DFB-like devices [8], while their electronically tunable counterparts, which are commonly preferred because of their higher tuning speed, have so far only shown comparatively poor performance [5], [9]. In this letter, we demonstrate for the first time state-of-the-art performance of electronically widely tunable DFB-type laser diodes. The presented sampled grating tunable twin-guide (SG-TTG) lasers fully cover a tuning range of over $40 \mathrm{~nm}$ with high sidemode suppression and large output power.

\section{DEVICE DESIGN AND FABRICATION}

The SG-TTG laser, as schematically depicted in Fig. 1, is based on the DFB-TTG laser [10]. In the SG-TTG laser [7], the vertically integrated tuning layer is longitudinally split into two sections, that contain sampled gratings (SGs) instead of DFB gratings. The sampling periods $\left(\Lambda_{S 1}\right.$ and $\left.\Lambda_{S 2}\right)$ of the SGs in the front and rear tuning section are slightly different. Thus, the comb-like reflection spectra of the SGs possess a slightly different periodicity, which permits the use of Vernier-effect tuning [11] to achieve wide quasi-continuous wavelength coverage. 
Whereas continuous tuning is obtained by tuning both reflectors simultaneously, large wavelength jumps, so-called supermode hops, are obtained by tuning one reflector while leaving the other one unchanged. Due to the vertical integration of active and tuning section, the laser behaves in essence like a DFB laser. Therefore, a phase tuning section is not necessary and only two tuning currents are required.

The devices were realized in a buried heterostructure design using the GaInAsP-InP material system. All four epitaxial steps were carried out in an Aixtron AIX 200/4 metal-organic vapor phase epitaxy system. The active region consists of a strained layer multi quantum well with an emission peak wavelength of $1.55 \mu \mathrm{m}$. The SGs were fabricated by a combination of holograhpy and optical lithography. The gratings with a pitch of $236 \mathrm{~nm}$ were etched into the 280-nm-thick tuning layer $\left(\lambda_{g}=\right.$ $1.40 \mu \mathrm{m})$. The effective coupling coefficient $\kappa_{\text {eff }}$ is estimated to be $\sim 10 \mathrm{~cm}^{-1}$. The $S G$ s have been designed to obtain a reflection peak spacing of 5.0 and $5.5 \mathrm{~nm}$ in the front and rear reflector, respectively.

The fabrication starts with the growth of the basic TTG structure. The last layer grown in this epitaxy step is the tuning layer, wherein then the gratings are defined. They are overgrown in the second epitaxy step by a thin $p$-InP cap. Subsequently, stripe mesas are etched by reative ion etching and laterally embedded with n-InP in a selective area epitaxy step. Finally, another selective area epitaxy step is carried out for the growth of the p-InP cladding. The devices are finalized by applying passivation and metallization layers.

Since facet reflections would interfere with the SG reflection spectrum and cause distortions, the front and back facets were formed by a combination of antireflection (AR) coatings and window structures [12] in order to obtain a facet reflectivity below $10^{-3}$. The $1200-\mu \mathrm{m}$-long and $1.3-\mu \mathrm{m}$-broad buried mesa was terminated approximately $30 \mu \mathrm{m}$ before the facet.

\section{DEVICE AND TUNing CHARACTERISTICS}

Characterization of the laser diodes was carried out at a heatsink temperature of $20{ }^{\circ} \mathrm{C}$. With tuning sections left unbiased, the devices reach the lasing threshold at an active region current of $27 \mathrm{~mA}$, corresponding to a current density of only $1.7 \mathrm{kA} / \mathrm{cm}^{2}$. The output power typically saturates at 20-25 $\mathrm{mW}$ due to the onset of leakage currents. Even higher output power levels should be obtainable by incorporation of blocking layers [13].

For the measurement of the tuning characteristics, the active region current was set to $250 \mathrm{~mA}$, slightly beyond the output power saturation point. Both tuning currents were swept in a nonlinear way up to a maximum current of $45 \mathrm{~mA}$. The wavelength map shown in Fig. 2 reveals the location of the various supermodes. Within these supermodes, the emission wavelength can be continuously and mode-hop-free tuned by up to $8.2 \mathrm{~nm}$. Only minor variations in the sidemode suppression ratio (SMSR) are observed during continuous tuning.

The emission wavelength as function of the rear reflector tuning setting was extracted from the wavelength map for the nine central supermodes and is depicted in Fig. 3. As can be seen, the spacing of the supermodes as well as the wavelength

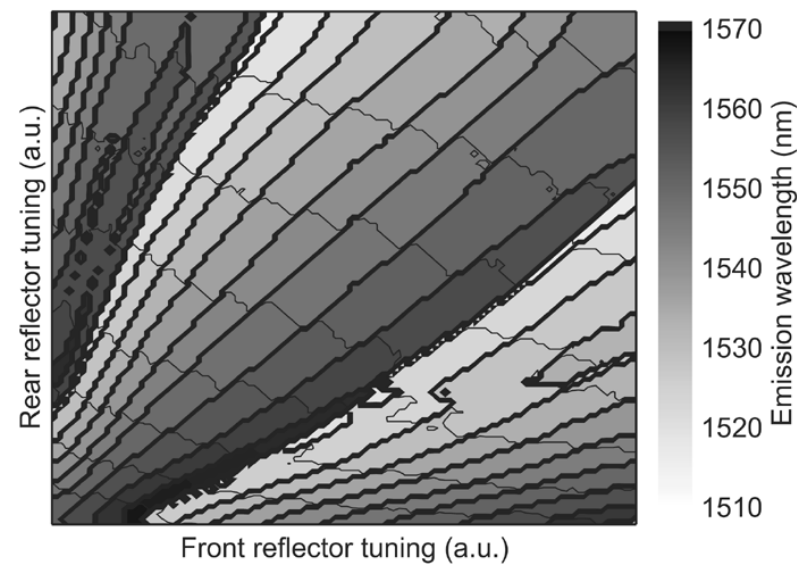

Fig. 2. Wavelength map of an SG-TTG laser diode. The various supermodes are enclosed by bold lines. Thin solid lines within the supermodes indicate iso-wavelength contours that are spaced in 1.0-nm intervals and are shown as a visual aid for determining the continuous tuning range of each supermode. Within the supermodes, continuous mode-hop free wavelength tuning of up to $8 \mathrm{~nm}$ is possible.

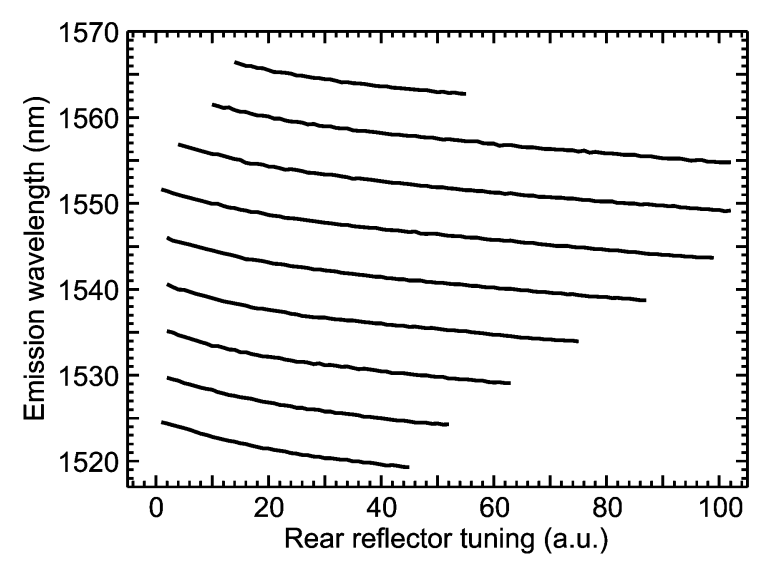

Fig. 3. Emission wavelength versus rear reflector tuning for the nine central supermodes of Fig. 2. Each supermode is unambiguously identified by the relationship between front and rear reflector tuning.

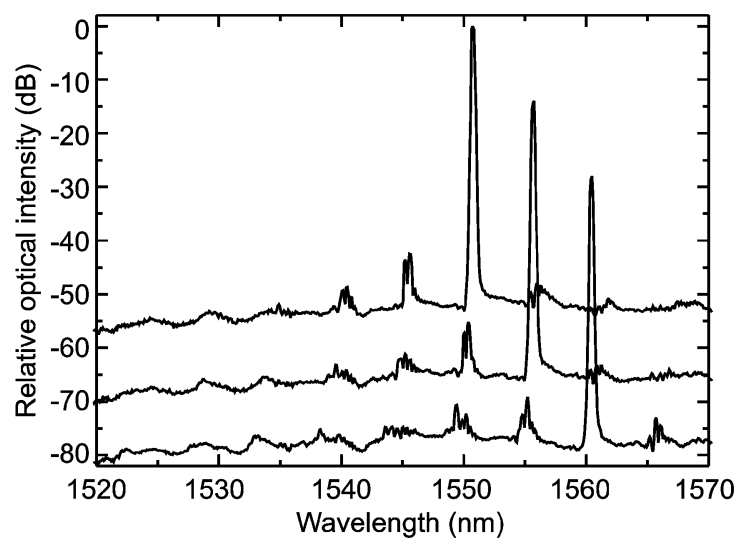

Fig. 4. Optical emission spectra of three neighboring supermodes, demonstrating the high spectral purity of the SG-TTG laser.

change within each of the supermodes is absolutely regular. Furthermore, the supermode spacing is also in good agreement with the SG design. These findings clearly show that spurious facet 


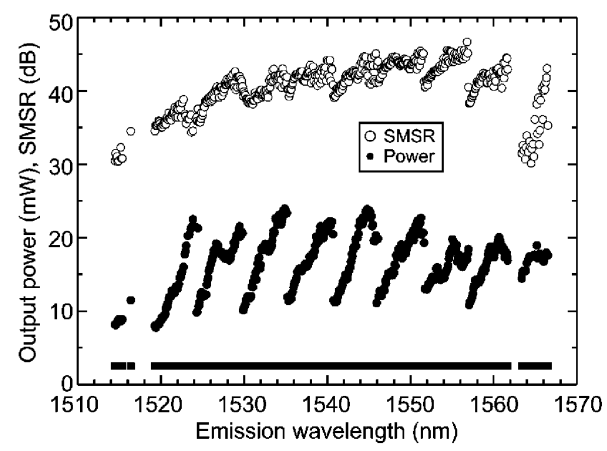

(a)

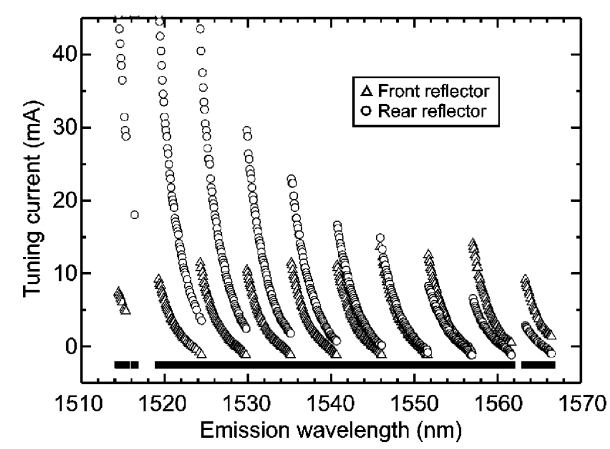

(b)

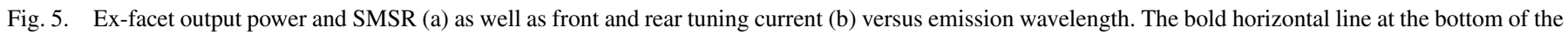

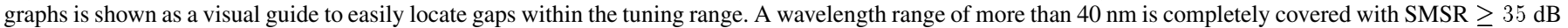
and output power $\geq 10 \mathrm{~mW}$.

reflections are effectively suppressed by the AR-coated window structures.

Optical emission spectra (Fig. 4) of three neighboring supermodes illustrate the high spectral purity of the SG-TTG laser. As usual for lasers employing Vernier-effect tuning, the SMSR is limited by reflections due to the nonvanishing overlap of neighboring SG reflection peaks and not by an adjacent cavity mode. Theoretically, a perfectly AR-coated DFB-laser exhibits two equally strong modes on either side of the stop-band. To lift this degeneracy, a distributed $\lambda / 4$-phase shift has been created in the center of some devices by narrowing the mesa width. However, experimentally it turned out that a phase shift is not essential to obtain longitudinal single-mode operation.

Output power, SMSR, and tuning currents as function of the emission wavelength are shown in Fig. 5. As can be seen, the SMSR is around $40 \mathrm{~dB}$ over most of the tuning range and remains above $30 \mathrm{~dB}$ even at the upper and lower limits of the tuning range. The output power varies between 8 and $24 \mathrm{~mW}$. Over a wavelength range of $41 \mathrm{~nm}$ (from 1520.5 to $1561.5 \mathrm{~nm}$ ), the SMSR and output power remain above $35 \mathrm{~dB}$ and $10 \mathrm{~mW}$, respectively.

With both tuning sections being left unbiased, lasing takes place at a wavelength of $1550 \mathrm{~nm}$. By tuning the rear and leaving the front tuning section unchanged, supermode hops take place toward shorter wavelength. Vice versa, supermode hops toward longer wavelength are obtained. The absolutely regular behavior of the tuning currents [Fig. 5(b)] again proves the regular tuning behavior of the SG-TTG. Due to the high tuning efficiency of the TTG laser, only small tuning currents are needed. For the present devices, a maximum tuning current of $45 \mathrm{~mA}$ is already sufficient to obtain tuning over a 40-50-nm wavelength range.

\section{CONCLUSION}

Sampled grating widely tunable twin-guide laser diodes, which fully cover a tuning range of more than $40 \mathrm{~nm}$ with high SMSR $(\geq 35 \mathrm{~dB})$ and large output power $(\geq 10 \mathrm{~mW})$, were presented. The SG-TTG laser requires only two tuning currents for wide quasi-continuous tuning and offers up to 8.2-nm broad continuous tuning regions. Hence, its characterization and control can be easy and fast.

\section{ACKNOWLEDGMENT}

The authors gratefully acknowledge the technical assistance of J. Grottenthaler, L. Mora, R. Heilmann, and E. Sckopke.

\section{REFERENCES}

[1] L. A. Coldren, G. A. Fish, Y. Akulova, J. S. Barton, and C. W. Coldren, "Tunable semiconductor lasers: A tutorial," J. Lightw. Technol., vol. 22, no. 1, pp. 193-202, Jan. 2004.

[2] J. Buus, M.-C. Amann, and D. J. Blumenthal, Tunable Laser Diodes and Related Optical Sources, 2nd ed. Piscataway, NJ: Wiley-IEEE Press, 2005.

[3] D. J. Robbins, G. Busico, L. Ponnampalam, J. P. Duck, P. J. Williams, R. A. Griffin, A. J. Ward, D. C. J. Reid, and K. Kasunic, "A high power, broad-band tuneable laser module based on a DS-DBR laser with integrated SOA," in Proc. Optical Fiber Conf. (OFC 2004), Los Angeles, CA, 2004, Paper TuE3, pp. 392-394.

[4] J. O. Wesström, G. Sarlet, S. Hammerfeldt, L. Lundqvist, P. Szabo, and P.-J Rigole, "State-of-the-art performance of widely tunable modulated grating Y-branch lasers," in Proc. Optical Fiber Conf. (OFC 2004), Los Angeles, CA, 2004, Paper TuE2, pp. 389-391.

[5] H. Ishii, Y. Kondo, F. Kano, and Y. Yoshikuni, "A tunable distributed amplification DFB laser diode (TDA-DFB-LD)," IEEE Photon. Technol. Lett., vol. 10, no. 1, pp. 30-32, Jan. 1998.

[6] S. Kim, Y. Chung, S.-H. Oh, and M.-H. Park, "Design and analysis of widely tunable sampled grating DFB laser diode integrated with sampled grating distributed Bragg reflector," IEEE Photon. Technol. Lett., vol. 16, no. 1, pp. 15-17, Jan. 2004.

[7] G. Morthier, B. Moeyersoon, and R. Baets, "A $\lambda / 4$-shifted sampled or superstructure grating widely tunable twin-guide laser," IEEE Photon. Technol. Lett., vol. 13, no. 10, pp. 1052-1054, Oct. 2001.

[8] R. Todt, T. Jacke, R. Meyer, and M.-C. Amann, "Thermally widely tunable laser diodes with distributed feedback," Appl. Phys. Lett., vol. 87, p. 021103, 2005.

[9] R. Todt, T. Jacke, R. Meyer, R. Laroy, G. Morthier, and M.-C. Amann, "Wide wavelength tuning of sampled grating tunable twin-guide laser diodes," Electron. Lett., vol. 40, pp. 1491-1492, Nov. 2004.

[10] M.-C. Amann, S. Illek, C. Schanen, and W. Thulke, "Tunable twin-guide laser: A novel laser diode with improved tuning performance," Appl. Phys. Lett., vol. 54, pp. 2532-2533, Jun. 1989.

[11] V. Jayaraman, Z.-M. Chuang, and L. A. Coldren, "Theory, design, and performance of extended tuning range semiconductor lasers with sampled gratings," IEEE J. Quantum Electron., vol. 29, no. 6, pp. 1824-1834, Jun. 1993.

[12] K. Utaka, S. Akiba, K. Sakai, and Y. Matsushima, "Effect of mirror facets on lasing characteristics of distributed feedback InGaAsP/InP laser diodes at $1.5 \mu \mathrm{m}$ range," IEEE J. Quantum Electron., vol. 20, no. 3, pp. 236-245, Mar. 1984.

[13] B. Schmidt, S. Illek, R. Gessner, and M.-C. Amann, "Design and realization of a buried-heterostructure tunable-twin-guide laser diode with electrical blocking regions," IEEE J. Quantum Electron., vol. 35, no. 5, pp. 794-802, May 1999. 